\title{
Alexander Potapov: "Neurosurgery uses advancements in modern biology in all of its aspects..."
}

\begin{abstract}
Alexander Potapov, laureate of the State Prize of the Russian Federation in Science and Technology for 2009, doctor of medical sciences, academician, Russian Academy of Medical Sciences, and deputy director of the N.N. Burdenko Scientific Research Institute of Neurosurgery of RAMS, talks to Acta Naturae about the achievements in modern neurosurgery, the latest developments in the field, and the close convergence between basic biology and clinical practice.
\end{abstract}

$\mathrm{A}$ lexander Alexandrovich, there has recently been such enormous progress in the field of neuroscience to the extent that some speak of a revolution in modern biology and medicine. What is the reason behind this trend?

The arsenal of the modern neurosurgeon has been supplemented by absolutely unique and constantly improving neuroimaging techniques. Above all, these include $\mathrm{X}$-ray computed and magnetic resonance tomography. Forty years ago, the brain in the skull was in a kind of "black box," and the surgeons could see what was happening inside only after trepanation. Now we can see all the happenings inside the brain of a living human by using quick, non-invasive techniques that are absolutely safe for the patient. Thanks to new neuroimaging methods, we are able to not only study the anatomy of the brain and the pathological formations, but also see the blood-flow and obtain quantative blood-flow data in milliliters per gram/minute for various structures and pathological formations in the brain. This allows to monitor the changes in the blood flow during the development of various pathological processes, as well as during their treatment. Methods allowing the mapping of metabolic changes in the brain are also gaining in popularity in clini- cal practice; these methods include positron-emission tomography and, in recent years, magnetic-resonance spectroscopy (essentially molecular neuroimaging). Using MRI spectroscopy, we can see the changes in tissue metabolism, such as alterations in the level of $\mathrm{N}$-acetylaspartate, which is a marker of neuron damage. These changes can be monitored not only in distinct areas, but also in whole sections of the brain at various levels. Neuroimaging methods allow doctors to distinguish cancerous processes from a disrupted blood-flow, as well as inflammatory or neurodegenerative processes. Modern neurovisualization methods are not only important for practical applications, but they are also a fundamental tool in research involving the study of the metabolic and structuro-functional changes during pathogenesis and neural tissue regeneration. These are fundamental issues in neurobiology.

Another possibility also recently developed and gaining wide application in practice is the ability to monitor a living brain in the process of various actions. This is called functional magnetic resonance tomography. For instance, we would ask a patient to do a task with his arm and then see what areas of the brain are activated, the cortical representation of the arm, and the location of any pathological formation, which can then be approached so as not to damage the functional structures of the brain. The possibilities of modern Diffusion-tensor MRI allow us to see the pathways of the brain. For instance, we can see the corticospinal tract, which transfers signals from the pyramidal cells of the cortex to the motor neurons of the spine, via long axons.

In your practice, you use the developments in various basic sciences; not only biology, but also physics, for example. Your collaboration with Vladislav Panchenko from the Institute of Laser and Informational Problems and Valery Chissov, the director of the P.A. Gertsin Moscow Oncological Scientific Research Institute, was awarded the Russian Federation State Prize in Science and Technology in 2009. Can you explain the essence of the new techniques that you developed and implemented?

Our collaboration with physicists proved very successful - our arsenal of tools was greatly improved through modern methods of rapid prototyping, which allow us to fully reproduce the exact copy of any human organ; body parts or the whole body. Currently, there are over a dozen prototyping methods. One of them allows the production of a full-scale copy of a virtual model from liquid polymers using a computer-controlled stereolithograph. There are other methods of rapid prototyping, such as powder 


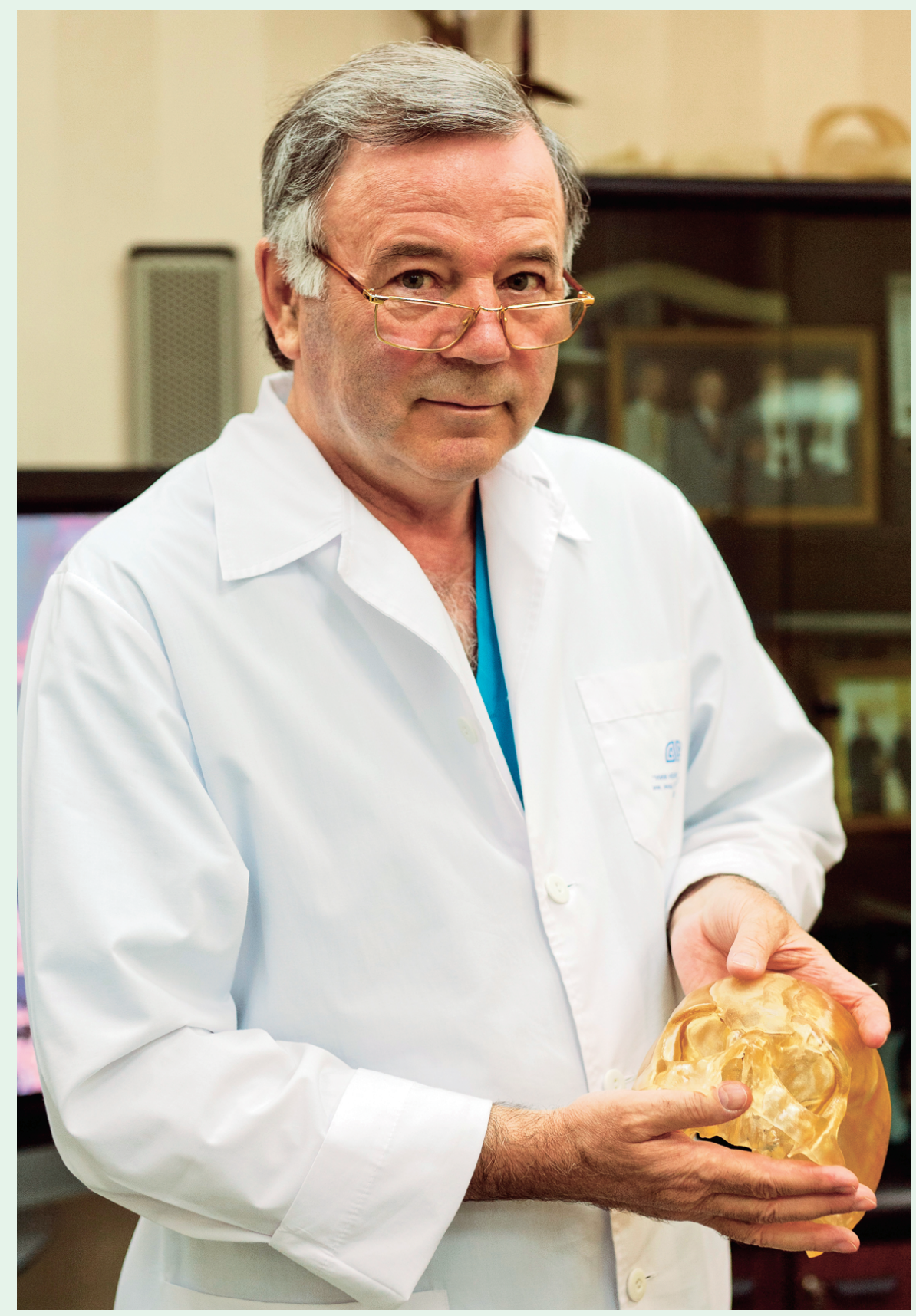

Alexander Potapov

metallurgy, which involves the use of metal powder, as well as the use of specialized laser technologies and a control computer to recreate complete copies of objects with very complex configurations.

Russian scientists have developed computer-controlled stereolithographs which provide the opportunity to recreate any object builds a solid copy of the damaged object out of the liquid polymer.

\section{How did all of this begin?}

During the 1990's, when we were just starting this project, it wasn't clear if any of the technology would be useful. We read publications about the methods involved in the identification of the remains of the Russian Royal Family. Scientists from the Institute of Laser and Informational Problems used stereolithography to create precise replicas of the discovered skulls and their fragments. We believed that this method could be used for reconstructive neurosurgery and quickly joined the development process. Our first copy-models were a bit rough, but as we streamlined the techniques, we found that this technology had huge potential for clinical practice.

What are the advantages of the rapid prototyping technique?

Complex defects and deformations of bone structures require various implants. Several tons of metal (particularly titanium) are being implanted into humans every year. Reconstructive spine operations involve artificial discs and stabilizing systems, which are prepared in advance. Then, during the operation, the surgeon selects one of the several available sizes so as to accommodate the needs of the patient at hand. So, rapid prototyping makes it possible to produce implants that suit each patient individually according to his or her real anatomy.

Suppose that a patient needs a right pelvic joint replacement. We make a three dimensional image of the left (healthy) pelvic joint and then use the modern methods of rapid prototyping to manufacture a pelvic joint implant which retains all the dimensions, peculiarities, and other anatomical traits of the original. The same is true for neurosurgery - if a person damages part of his or her face, then the plastic surgeon could restore that part of the face by us- 
ing the undamaged healthy region as a guide. We obtain a virtual $3 \mathrm{D}$ image of the skull, then take the undamaged portion, reflect it and place it into the damaged portion. In the end, we have a fully restored and completely symmetrical skull. If the facial damage is in the center of the face, we use a virtual 3D "donor" image and restore the missing central part. Over many years of using computer tomography, a huge database of images has been accumulated that can be used to virtually choose the appropriate configuration for the organ in question.

Rapid prototyping techniques are being developed in other countries, such as Japan, the U.S., and Germany. Yet your technology has already been successfully implemented in 50 medical centers. Why is our Russian technology competitive?

There are companies that manufacture similar custom-made implants. However, our technique is different in that it involves the surgeon in the implant manufacturing process. However, the surgeon's main task is not only to restore the bone structure, but also to reconstruct the nervous tissue and individual neurons.

So the saying "nervous cells do not regenerate" is a misconception?

The thesis that a nervous cell cannot regenerate, postulated by the Nobel Prize laureate Santiago Ramón y Cajal, has been revised. Collaboration with the other biologists involved in the study of the problems of reconstructing the nerve cell structure will allow us to transfer the achievements in experimental neurobiology into clinical practice as soon as possible.

There are currently several approaches that can facilitate the regeneration of nervous cells. There are several known neurotrophic factors that can stimulate the cell's regenerative processes. Lithium, one of the simplest elements, has been shown to promote regeneration. There are also various compounds that inhibit the enzymes that block neural regeneration. Experiments have shown that neurons can be regenerated by neurotransplantation. However, for clinical use, we need more progress in the field of neurobiology. Then we will be able to use these discoveries in clinical practice.

So, you mean to say that medical practice and fundamental biology are integrated much more closely than we think?

Neurosurgery uses advancements in modern biology in all of its aspects. For instance, when we study the processes of brain damage caused by a stroke, trauma or cancerous damage, we are also studying the biology of these processes. How does this process progress in the neural tissue? Which mechanisms can lead to regeneration, and which can lead to cell death? Therefore, we need to know the biology of these processes. What action should we take to restore the damaged neural tissue, and all of its elements - neurons, the glial complex, and the vascular network? How do compounds in the blood reach their final target (brain cells) and overcome the unique hematoencephalic barrier? Today we can manage these processes in order to achieve a therapeutic effect.

Genetic research is also very important to us. Modern knowledge shows that the brain's reaction to damage is determined not only by the type of damage per se, but also by the genetic traits of the individual, i.e. genetic profile. There is evidence that genetic polymorphism in a number of genes determines the strength of the response of neural tissue to damage. Any damage leads to a universal reaction - inflammation. In neural tissue, this is neuroinflammation, which is manifested by increased hematoencephalic barrier permeability, edema (intracellular, intercellular, and mixed), disrupted circulation of liquor and blood, and other general metabolic disorders. The nature of these reactions and the following regenerative processes may be determined by the peculiarities of the human genome, or by the so-called genetic polymorphism. One of the issues of your journal published a review by Professor V.S. Baranov (Editor's note - see Acta Naturae, v.1, №3, 2010) on genetic polymorphism and the prospects of medical genomics. This field is also very important for us, clinicians, since it means that our prognoses on the course of the pathological process and its outcomes should factor in not only the etiology, pathogenesis and clinical picture of the disease, but also the genetic profile of the patient, including his or her risk factors, which can lead to various scenarios of disease development.

And what about the reverse process - which methods of clinical medicine are used for studying the more basic problems?

The possibilities of modern neuroimaging techniques, which we discussed earlier, allow the study of more fundamental issues - the relationship between the structure and function of the brain. For instance, how do the brains of different people (lefties, righties, people with left or right-hemisphere dominance, or no dominance at all) function after the appearance of a pathological area? How does the brain adapt to aging, development, and pathological effects? These are all basic issues concerning the functioning of the nervous system (and the brain in particular) under normal and pathological conditions. Today these methods, which are becoming increasingly useful in clinical practice, are also successfully being used in basic neurobiological research- the study of the brain and the nervous system.

Interview by Elena Novoselova 